\title{
Wave diffraction from a truncated cylinder with an upper porous sidewall and an inner column
}

\author{
De-Zhi Ning ${ }^{* 1}$, Xuan-Lie Zhao ${ }^{1}$, Bin Teng ${ }^{1}$, Lars Johanning ${ }^{1,2}$
}

\begin{abstract}
An analytical model based on linear potential theory is proposed to predict the three-dimensional wave diffraction from a truncated cylinder with an upper porous sidewall and an inner column in the finite water depth. The velocity potential is analytically derived in the whole fluid domain based on the method of variable separation and eigen-function expansion technique. The continuous conditions of pressure and velocity potential are satisfied on the interface between the adjacent sub-domains. Wave forces are calculated directly from the incident and diffracted potentials. The model is validated in comparison with other published results of wave diffraction from a porous bottom-mounted cylinder and impermeable truncated cylinder, respectively. Then the numerical tests are performed to investigate the effects of the porous coefficient $G$, the draft ratio $h / h_{1}$ ( $h$ and $h_{1}$ mean the drafts of the porous part and whole cylinder, respectively), the ratio of the inner and outer radii $b / a$ and the water depth $d / h_{1}(d$ means the water depth) on the wave forces acting on the structure. It is found that, by introducing an upper porous sidewall, the hydrodynamic loads are improved in comparison with the fully impermeable structure, which may be benefit to enhance the survivability of the relating marine structure.
\end{abstract}

Keywords: Potential theory; wave diffraction; analytical derivation; porous structure; truncated cylinder 


\section{Introduction}

Wave exciting forces are key elements to be considered for the safe operation of marine structures, such as breakwaters, wave power devices and offshore platforms (Hirdaris et al., 2014). Effective optimization of the wave loads on the structure may lead to the reduction in the cost of offshore structures. Structures with porous portion constitute an important class of maritime structures. By comparing with the impermeable structure, wave force acting on the porous structures is relatively reduced and the wave reflection is decreased, thus the porous structure is favored while the wave force reduction or excellent wave attenuation performance is needed (Chwang and Chan, 2003; Chandrasekaran et al., 2015; Teng et al., 2000).

There has been a great deal of effort directed towards quantifying wave interactions with porous ocean structures. Generally, the porous structures may be divided into two categories, i.e., bottom-mounted structures and truncated structures. The former was mainly focused on the porous breakwaters and bottom-mounted cylinders. The use of porous structures as breakwaters was extensively investigated both theoretically and experimentally (Jarlan, 1961; Yu and Chwang, 1994; Hu et al., 2002; Williams and Li, 1998; Wang and Ren, 1993; Yu, 1995; Liu et al., 2006; 2008). A detailed review of the studies on the interaction between waves and perforated breakwaters can be found in Huang et al. (2011). Three-dimensional (3-D) wave diffraction from an array of bottom-mounted cylinders with porous sidewalls were investigated by Williams and Li (2000), Park et al. (2014), Sankarbabu et al. (2007) and Li et al. (2004). It was found that the porous sidewall can significantly reduce both the hydrodynamic loads experienced by the cylinders and the wave run-up. Mandal and Sahoo (2015) dealt with the hydroelastic problem of concentric flexible porous cylinder systems in two-layer fluid. The results showed that the full wave reflections in the surface and internal modes may occur for some special cases. However, the investigation of perforation in the truncated structures is still quite rare up to now. Williams et al. (2000) theoretically studied the wave diffraction and radiation from a floating cylinder whose middle part is porous and found that the porous part of the structure has a significant influence on the horizontal hydrodynamic force. However, its influence on the vertical force is relatively weak. Recently, the hydrodynamic characteristics of the porous structures used as the bottom mounted offshore platform and tension leg platform were investigated theoretically (Lee 
and Ker, 2002; Chandrasekaran and Sharma, 2010; Chandrasekaran et al., 2015).

The porous materials as ocean structures can reduce the wave exciting force, which has been testified in the previous studies (Chandrasekaran et al., 2015). Since the most wave energy propagates nearby the free-surface, a porous sidewall surrounding an inner column as the top part of a truncated structure to dissipate energy (see Fig. 1) is expected to have the potential to efficiently reduce the wave loads. The proposed structure (see Fig. 1) can be regarded as the refit of the traditional impermeable structure or the compound structure. To the authors' best knowledge, there is little research to study the hydrodynamic characteristic of such type of structures. The proposed structure is expected to improve the hydrodynamics in comparison with the impermeable truncated structures. The latters have been extensively investigated by Wolgamot et al. (2012), Göteman et al. (2014), Zheng and Zhang (2015), Kara (2016), Ning et al. (2016), Johanning (2009) and Johanning et al. (2001). This paper aims to study the diffraction problem of a truncated circular cylinder with top porous sidewall and to quantify the effects of various wave and structural parameters on the wave loads. Analytical solution based on linear potential flow theory for wave diffraction from such a truncated cylinder is derived using the eigen-function expansion approach. Different from the analytical solutions of hydrodynamic problem of the general truncated cylinder with porous part (Williams et al., 2000; Lee and Ker, 2002), the effect of the imaginary part of the porous coefficient is considered and the present model can conveniently deal with the diffraction problem of truncated cylinder and the bottom-mounted cylinder with the surface-piercing porous part.

The paper is organized as follows. In Section 2, the governing equation and boundary conditions are described. In Section 3, the analytical derivation of the diffraction problem is given. In Section 4, the results are presented and discussed. Finally, the conclusions are presented in Section 5 .

\section{Theoretical formulation}

The problem of wave diffraction from a truncated circular cylinder with an upper porous sidewall outside an inner cylinder is considered as shown in Fig. 1. Symbols $a, b, h, h_{1}$ and $d$ represent outer cylinder radius, inner cylinder radius, draft of the upper porous part, draft of the whole cylinder and the static water depth, respectively. A cylindrical polar coordinate $(r, \theta, z)$, 
combined with a Cartesian coordinate $(x, y, z)$, is built with its origin located at the center of the cylinder on the still-water level.
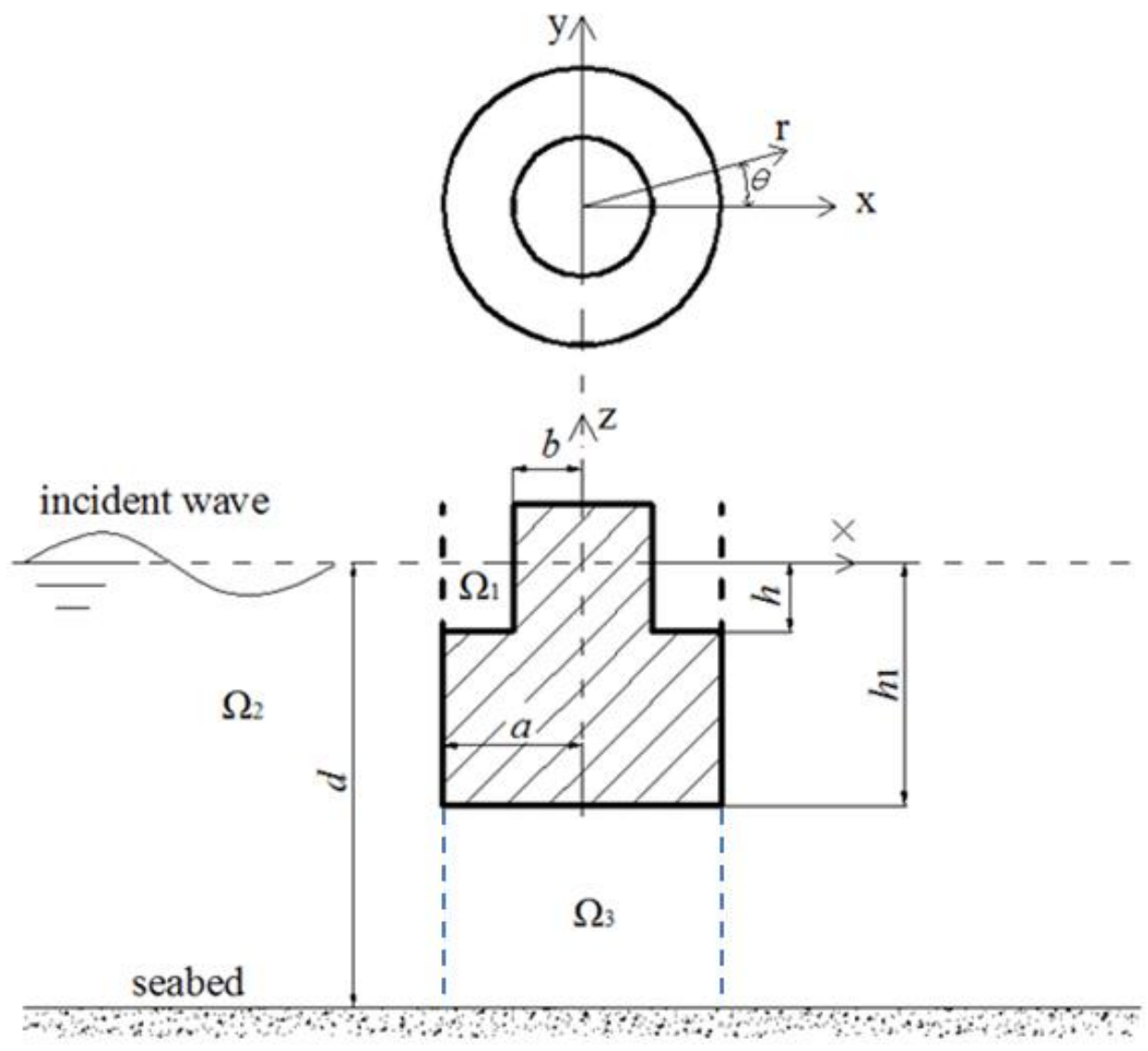

Figure 1 Definition sketch

The structure is subjected to regular surface waves propagating in the positive $x$-direction with a wave height $H(H=2 A$, where $A$ is wave amplitude) and an angular frequency $\omega$. Under the frame of linear potential theory, the fluid can be described in terms of a complex velocity potential $\Phi(r, \theta, z, t)=\operatorname{Re}\left[\phi(r, \theta, z) e^{-\mathrm{i} \omega t}\right]$, where $\operatorname{Re}$ denotes the real part of a complex expression, i the imaginary unit and $\phi(r, \theta, z)$ represents the spatial potential. Subsequently, the common time-dependent term $e^{-\mathrm{i} \omega t}$ can be dropped from all the dynamic variables.

As shown in Fig. 1, the fluid domain is divided into three regions: an interior region defined by $\Omega_{1}(-h \leq \mathrm{z} \leq 0, b \leq r \leq a)$; an exterior region defined by $\Omega_{2}(-d \leq \mathrm{z} \leq 0, r \geq a)$ and another region beneath the cylinder, i.e., $\Omega_{3}\left(-d \leq \mathrm{z} \leq-h_{1}, 0 \leq r \leq a\right)$. Since the present study is solved in the frequency domain, the velocity potential in each region $\Omega_{j}$ is denoted by $\phi_{j}, j=1,2$ and 3 . Each potential satisfies Laplace equation in the corresponding flow region, namely 


$$
\nabla^{2} \phi_{j}=0 \text { for } j=1,2,3 \text { in } \Omega_{j}
$$

The potential also satisfies the appropriate boundary conditions on the free-surface, and sea-bed, namely

$$
\begin{gathered}
\frac{\partial \phi_{j}}{\partial z}=\frac{\omega^{2}}{g} \phi_{j} \text { for } z=0, j=1,2,3 \\
\frac{\partial \phi_{j}}{\partial z}=0 \text { for } z=-d, j=2,3
\end{gathered}
$$

where $g$ is the acceleration due to gravity.

The boundary condition on the impermeable surface of cylinder can be expressed as

$$
\begin{aligned}
& \frac{\partial \phi_{1}}{\partial z}=0 \text { for } b<r<a, z=-h \\
& \frac{\partial \phi_{3}}{\partial z}=0 \text { for } 0<r<a, z=-h_{1} \\
& \frac{\partial \phi_{1}}{\partial r}=0 \text { for } r=b,-h<z<0 \\
& \frac{\partial \phi_{2}}{\partial r}=0 \text { for } r=a,-h_{1}<z<-h
\end{aligned}
$$

The boundary condition on the porous circular wall can be expressed as follows (Sollitt and Cross, 1972; Yu, 1995):

where $k$ is the wave number,

$$
\frac{\partial \phi_{1}}{\partial r}=\mathrm{i} k G\left(\phi_{1}-\phi_{2}\right) \text { for } \quad r=a,-h<z<0
$$

porous wall (the thickness is negligible geometrically) and $\gamma, f$ and $C_{m}$ are the porosity, the linearized resistance coefficient and the added-mass coefficient of the porous medium, respectively (Yu, 1995). The parameter $G$ can be write as $G_{r}+\mathrm{i} G_{i}$, where $G_{r}$ denotes the real part and $G_{i}$ the imaginary part. Physically, $G_{r}$ and $G_{i}$ represent the drag term and the inertia term, which lead to the wave energy loss and the phase change, respectively (Teng et al., 2000; Teng et al., 2001).

On the cylindrical surface of $r=a$, the potentials should satisfy the following matching 
conditions:

$$
\begin{gathered}
\phi_{2}=\phi_{3} \text { for } r=a,-d<z<-h_{1} \\
\frac{\partial \phi_{2}}{\partial r}=\left\{\begin{array}{c}
\frac{\partial \phi_{1}}{\partial r} \text { for } r=a,-h<z<0 \\
0 \text { for } r=a,-h_{1}<z<-h \\
\frac{\partial \phi_{3}}{\partial r} \text { for } r=a,-d<z<-h_{1}
\end{array}\right.
\end{gathered}
$$

The velocity potentials in the flow region consist of the incident potential and diffracted potential. At infinity, the diffracted component $\left(\phi_{d}\right)$ of the velocity potential in the exterior region must satisfy the infinite condition as follows

$$
\lim _{r \rightarrow \infty}\left[\sqrt{r}\left(\frac{\partial \phi_{d}}{\partial r}-\mathrm{i} k \phi_{d}\right)\right]=0
$$

\section{Analytical derivation}

In this section, an analytical solution of the wave diffraction problem is derived. In domain $\Omega_{2}$, the potential $\phi_{2}$ can be written in terms of the following well-known eigen-function expansion:

$$
\phi_{2}=\phi_{i}+\phi_{d}=-\frac{\mathrm{i} g A}{\omega}\left(Z_{0}\left(k_{0} z\right) \sum_{m=0}^{\infty} \varepsilon_{m} \mathrm{i}^{m} \mathrm{~J}_{m}\left(k_{0} r\right) \cos m \theta+\sum_{m=0}^{\infty} \varepsilon_{m} \mathrm{i}^{m} \cos m \theta \sum_{i=0}^{\infty} A_{m i} Z_{i}\left(k_{i} z\right) U_{m}\left(k_{i} r\right)\right)
$$

where $\varepsilon_{0}=1$ and $\varepsilon_{m}=2(m>1), A_{m i}(m, i=0,1,2 \ldots)$ are unknown coefficients, $\mathbf{J}_{m}\left(k_{0} r\right)$ denotes the first kind of Bessel function of order $m$. Wave number $k_{0}$ and $k_{i}(i=1,2,3 \ldots)$ can be derived by the following dispersion relation.

$$
\omega^{2}= \begin{cases}g k_{i} \tanh k_{i} d & i=0 \\ -g k_{i} \tan k_{i} d & i \geq 1\end{cases}
$$

The radial eigen-functions $U_{m}\left(k_{i} r\right)$ are given as follows

$$
U_{m}\left(k_{i} r\right)=\left\{\begin{array}{cc}
\mathrm{H}_{m}^{(1)}\left(k_{i} r\right) / \mathrm{H}_{m}^{(1)}\left(k_{i} a\right) & i=0 \\
\mathrm{~K}_{m}\left(k_{i} r\right) / \mathrm{K}_{m}\left(k_{i} a\right) & i \geq 1
\end{array}\right.
$$

where $\mathrm{H}_{m}^{(1)}\left(k_{i} r\right)$ denotes the first kind of Hankel function of order $m$; and $\mathrm{K}_{m}\left(k_{i} r\right)$ is the second kind of modified Bessel function of order $m$. The vertical eigen-functions $Z_{i}\left(k_{i} z\right)$ form an orthogonal set in $[-d, 0]$ defined as 


$$
Z_{i}\left(k_{i} z\right)=\left\{\begin{array}{cc}
\cosh k_{i}(z+d) / \cosh k_{i} d & i=0 \\
\cos k_{i}(z+d) / \cos k_{i} d & i \geq 1
\end{array}\right.
$$

The scattered velocity potential $\phi_{1}$ in domain $\Omega_{1}$, which satisfies the appropriate free-surface and body boundary conditions, can be written as

$$
\phi_{1}=-\frac{\mathrm{i} g A}{\omega} \sum_{m=0}^{\infty} \varepsilon_{m} \mathrm{i}^{m} \cos m \theta \sum_{j=0}^{\infty} Y_{j}\left(\lambda_{j} z\right)\left[C_{m j} V_{m}^{(1)}\left(\lambda_{j} r\right)+D_{m j} V_{m}^{(2)}\left(\lambda_{j} r\right)\right]
$$

where $\lambda_{j}$ can be derived by the following relations

$$
\omega^{2}= \begin{cases}g \lambda_{j} \tanh \lambda_{j} h & j=0 \\ -g \lambda_{j} \tan \lambda_{j} h & j \geq 1\end{cases}
$$

The radial eigen-functions $V_{m}^{(1)}\left(\lambda_{j} r\right)$ and $V_{m}^{(2)}\left(\lambda_{j} r\right)$ are given by

$$
V_{m}^{(1)}\left(\lambda_{j} r\right)=\left\{\begin{array}{cc}
\mathrm{H}_{m}^{(1)}\left(\lambda_{j} r\right) / \mathrm{H}_{m}^{(1)}\left(\lambda_{j} a\right) & j=0 \\
\mathrm{I}_{m}\left(\lambda_{j} r\right) / \mathrm{I}_{m}\left(\lambda_{j} a\right) & j \geq 1
\end{array} ; \quad V_{m}^{(2)}\left(\lambda_{j} r\right)=\left\{\begin{array}{cc}
\mathrm{H}_{m}^{(2)}\left(\lambda_{j} r\right) / \mathrm{H}_{m}^{(2)}\left(\lambda_{j} a\right) & j=0 \\
\mathrm{~K}_{m}\left(\lambda_{j} r\right) / \mathrm{K}_{m}\left(\lambda_{j} a\right) & j \geq 1
\end{array}\right.\right.
$$

where $\mathbf{H}_{m}^{(2)}\left(\lambda_{j} r\right)$ denotes the second kind of Hankel function of order $m, \mathbf{I}_{m}\left(\lambda_{j} r\right)$ the first kind of modified Bessel function of order $m$.

The vertical eigen-functions $Y_{j}\left(\lambda_{j} z\right)$ form an orthogonal set in $[-h, 0]$ defined as

$$
Y_{j}\left(\lambda_{j} z\right)=\left\{\begin{array}{cc}
\cosh \lambda_{j}(z+h) / \cosh \lambda_{j} h \quad j=0 \\
\cos \lambda_{j}(z+h) / \cos \lambda_{j} h \quad j \geq 1
\end{array}\right.
$$

The potentials in region 1 satisfying boundary condition (6) can be re-written as

$$
\phi_{1}=-\frac{\mathrm{i} g A}{\omega} \sum_{m=0}^{\infty} \varepsilon_{m} i^{m} \cos m \theta \sum_{j=0}^{\infty} C_{m j} Y_{j}\left(\lambda_{j} z\right) F_{m}\left(\lambda_{j} r\right)
$$

where

$$
F_{m}\left(\lambda_{j} r\right)=V_{m}^{(1)}\left(\lambda_{j} r\right)-\frac{V_{m}^{(1)}{ }^{\prime}\left(\lambda_{j} b\right)}{V_{m}^{(2)}{ }^{\prime}\left(\lambda_{j} b\right)} V_{m}^{(2)}\left(\lambda_{j} r\right)
$$

where $V_{m}^{(1)}{ }^{\prime}\left(\lambda_{j} b\right)=\left.\frac{\partial V_{m}^{(1)}\left(\lambda_{j} r\right)}{\partial r}\right|_{r=b}, V_{m}^{(2)}{ }^{\prime}\left(\lambda_{j} b\right)=\left.\frac{\partial V_{m}^{(2)}\left(\lambda_{j} r\right)}{\partial r}\right|_{r=b}$.

The velocity potential $\phi_{3}$ in region 3 , which satisfies the sea-bed and structural boundary conditions (i.e., Eqs. (3) and (5)), is given as 


$$
\phi_{3}=-\frac{\mathrm{i} g A}{\omega} \sum_{m=0}^{\infty} \varepsilon_{m} \mathrm{i}^{m} \cos m \theta \sum_{j=0}^{\infty} B_{m j} Q_{j}\left(\mu_{j} z\right) W_{m}\left(\mu_{j} r\right)
$$

where $\mu_{j}=j \pi /\left(d-h_{1}\right)$, the radial eigen-functions $W_{m}\left(\mu_{j} r\right)$ are given as

$$
W_{m}\left(\mu_{j} r\right)=\left\{\begin{array}{c}
(r / a)^{m} \quad j=0 \\
\mathrm{I}_{m}\left(\mu_{j} r\right) / \mathrm{I}_{m}\left(\mu_{j} a\right) \quad j \geq 1
\end{array}\right.
$$

The vertical eigen-functions $Q_{j}\left(\mu_{j} z\right)$ form an orthogonal set in [- $\left.d,-h_{1}\right]$ defined as

$$
Q_{j}\left(\mu_{j} z\right)=\left\{\begin{array}{c}
\frac{\sqrt{2}}{2} \quad j=0 \\
\cos \mu_{j}(z+d) \quad j \geq 1
\end{array}\right.
$$

Based on the continuity equations (i.e., Eqs. (8-10)), three set of algebraic equations can be formed by using the orthogonal properties of the vertical eigen-functions in each fluid region

$$
\begin{gathered}
\int_{-h}^{0} \frac{\partial \phi_{1}}{\partial r} Y_{j}\left(\lambda_{j} z\right) \mathrm{d} z=\int_{-h}^{0} \mathrm{i} k G\left(\phi_{1}-\phi_{2}\right) Y_{j}\left(\lambda_{j} z\right) \mathrm{d} z \text { for } r=a \\
\int_{-d}^{-h_{1}} \phi_{2} Q_{j}\left(\mu_{j} z\right) \mathrm{d} z=\int_{-d}^{-h_{1}} \phi_{3} Q_{j}\left(\mu_{j} z\right) \mathrm{d} z \quad \text { for } r=a \\
\int_{-d}^{0} \frac{\partial \phi_{2}}{\partial r} Z_{i}\left(k_{i} z\right) \mathrm{d} z=\int_{-h}^{0} \frac{\partial \phi_{1}}{\partial r} Z_{i}\left(k_{i} z\right) \mathrm{d} z+\int_{-d}^{-h_{1}} \frac{\partial \phi_{3}}{\partial r} Z_{i}\left(k_{i} z\right) \mathrm{d} z \text { for } r=a
\end{gathered}
$$

The sets of algebraic equations for the potential coefficients can be solved by the standard matrix techniques. And the unknown potential coefficients $A_{m i}, B_{m j}$ and $C_{m j}$ are determined by truncating the infinite series in Eqs. (12), (14) and (15) to a finite terms firstly. Thus, the potential in each fluid region can be uniquely determined.

Once the velocity potential in each region is obtained, the hydrodynamic forces in the $x$ and $z$ directions and the overturning moment about $y$ axis through the rotational center $\left(0,0, z_{0}\right)$, may be calculated by integrating the pressure over the average wet cylinder surface.

The total horizontal force can be separated into three parts:

$$
F_{\mathrm{x}}=F_{\mathrm{x} 1}+F_{\mathrm{x} 2}+F_{\mathrm{x} 3}
$$

in which the horizontal force on surface $S_{\mathrm{b} 1}(-h \leq \mathrm{z} \leq 0, r=b)$ is written as

$$
F_{\mathrm{x} 1}=\iint_{S_{\mathrm{b} 1}} p n_{\mathrm{x}} \mathrm{d} s=\mathrm{i} \omega \rho \int_{-h}^{0} \int_{0}^{2 \pi} \phi_{1}(-\cos \theta) \mathrm{d} z \mathrm{~d} \theta=-2 \pi \mathrm{i} \rho g A b \sum_{j=0}^{J} C_{1 j} F_{1}\left(\lambda_{j} b\right) \int_{-h}^{0} Y_{j}\left(\lambda_{j} z\right) \mathrm{d} z
$$

the horizontal force on surface $S_{\mathrm{b} 2}\left(-h \leq \mathrm{z} \leq 0, r=a^{-}\right)$is given as 


$$
F_{\mathrm{x} 2}=\iint_{S_{b 2}} p n_{\mathrm{x}} \mathrm{d} s=\mathrm{i} \omega \rho \int_{-h}^{0} \int_{0}^{2 \pi} \phi_{1} \cos \theta \mathrm{d} z \mathrm{~d} \theta=2 \pi \mathrm{i} \rho g A a \sum_{j=0}^{J} C_{1 j} F_{1}\left(\lambda_{j} a\right) \int_{-h}^{0} Y_{j}\left(\lambda_{j} z\right) \mathrm{d} z
$$

$$
\begin{aligned}
F_{\mathrm{x} 3} & =\iint_{S_{b 3}} p n_{\mathrm{x}} \mathrm{d} s=\mathrm{i} \omega \rho \int_{-h_{1}}^{0} \int_{0}^{2 \pi} \phi_{2}(-\cos \theta) \mathrm{d} z \mathrm{~d} \theta \\
& =-2 \pi \mathrm{i} \rho g A a\left\{J_{1}\left(k_{0} a\right) \int_{-h_{1}}^{0} Z_{0}\left(k_{0} z\right) \mathrm{d} z+\sum_{i=0}^{I} A_{1 i} U_{i}\left(k_{i} a\right) \int_{-h_{1}}^{0} Z_{i}\left(k_{i} z\right) \mathrm{d} z\right\}
\end{aligned}
$$

The total vertical force $F_{\mathrm{Z}}$ can be written as the sum of forces on surfaces $S_{\mathrm{h} 1}(b \leq r \leq a, z=$ $-h)$ and $S_{\mathrm{h} 2}\left(0 \leq r \leq a, z=-h_{1}\right)$ :

$$
\begin{aligned}
& F_{\mathrm{z}}=\iint_{S_{\mathrm{h} 2}} p n_{\mathrm{z}} \mathrm{d} s-\iint_{S_{\mathrm{h} 1}} p n_{\mathrm{z}} \mathrm{d} s=\mathrm{i} \omega \rho \int_{0}^{a} \int_{0}^{2 \pi} \phi_{3}\left(r,-h_{1}, \theta\right) r \mathrm{~d} r \mathrm{~d} \theta-\mathrm{i} \omega \rho \int_{b}^{a} \int_{0}^{2 \pi} \phi_{1}(r,-h, \theta) r \mathrm{~d} r \mathrm{~d} \theta \\
& =2 \pi \rho g A \sum_{i=0}^{\infty} B_{0 j} Q_{j}\left(\mu_{j}\left(-h_{1}\right)\right) \int_{0}^{a} W_{0}\left(\mu_{j} r\right) r \mathrm{~d} r-2 \pi \rho g A \sum_{j=0}^{\infty} C_{0 j} Y_{j}\left(\lambda_{j}(-h)\right) \int_{0}^{a} F_{0}\left(\lambda_{j} r\right) r \mathrm{~d} r
\end{aligned}
$$

The moment $M_{y}$ on the cylinder consists of two parts:

$$
M_{\mathrm{y}}=\iint_{s} p\left[\left(z-z_{0}\right) n_{x}-x n_{z}\right] \mathrm{d} s=M_{\mathrm{y} 1}+M_{\mathrm{y} 2}
$$

where $M_{\mathrm{y} 1}$ and $M_{\mathrm{y} 2}$ are the moment induced by the horizontal force and vertical force, respectively. The $M_{\mathrm{y} 1}$ can be separated into three parts:

$$
M_{\mathrm{y} 1}=M_{\mathrm{y} 1 \_1}+M_{\mathrm{y} 1 \_2}+M_{\mathrm{y} 1 \_3}
$$

where $M_{\mathrm{y} 1}$ refers to the sum of the moment induced by the force on the region of $S_{\mathrm{b} 1}(-h<z<0, r$ $=b), S_{\mathrm{b} 2}\left(-h<z<0, r=a^{-}\right)$and $S_{\mathrm{b} 3}\left(-h_{1}<z<0, r=a^{+}\right)$, namely, $M_{\mathrm{y} 1 \_1}, M_{\mathrm{y} 1 \_2}$ and $M_{\mathrm{y} 1 \_3}$.

$$
\begin{aligned}
& M_{\mathrm{y} 1 \_1}=\mathrm{i} \omega \rho \int_{-h}^{0} \int_{0}^{2 \pi}\left[b \phi_{1}(b, \theta, z)\left(z-z_{0}\right)(-\cos \theta)\right] \mathrm{d} \theta \mathrm{d} z \\
& =-2 \pi \mathrm{i} \rho g A b C_{1 j} F_{1}\left(\lambda_{j} b\right) \int_{-h}^{0} Y_{j}\left(\lambda_{j} z\right)\left(z-z_{0}\right) \mathrm{d} z \\
& M_{\mathrm{y} 1 \_2}=-\mathrm{i} \omega \rho \int_{-h}^{0} \int_{0}^{2 \pi}\left[a \phi_{1}(a, \theta, z)\left(z-z_{0}\right)(-\cos \theta)\right] \mathrm{d} \theta \mathrm{d} z \\
& =2 \pi \mathrm{i} \rho g A a C_{1 j} F_{1}\left(\lambda_{j} a\right) \int_{-h}^{0} Y_{j}\left(\lambda_{j} z\right)\left(z-z_{0}\right) \mathrm{d} z \\
& M_{\mathrm{y}{ }_{-3}}=\mathrm{i} \omega \rho \int_{-h_{1}}^{0} \int_{0}^{2 \pi}\left[a \phi_{2}(a, \theta, z)\left(z-z_{0}\right)(-\cos \theta)\right] \mathrm{d} \theta \mathrm{d} z \\
& =-2 \pi \mathrm{i} \rho g A a\left\{\mathrm{~J}_{1}\left(k_{0} a\right) \int_{-h_{1}}^{0} Z_{0}\left(k_{0} z\right)\left(z-z_{0}\right) \mathrm{d} z+\sum_{i=1}^{I} A_{1 i} U_{i}\left(k_{i} a\right) \int_{-h_{1}}^{0} Z_{i}\left(k_{i} z\right)\left(z-z_{0}\right) \mathrm{d} z\right\}
\end{aligned}
$$

$M_{\mathrm{y} 2}$ can be separated into two parts: 
where $M_{\mathrm{y} 2}$ refers to sum of the moment induced by the force on the surfaces $S_{\mathrm{h} 1}(b<r<a, z=-h)$ and $S_{\mathrm{h} 2}\left(0<r<a, z=-h_{1}\right)$, namely, $M_{\mathrm{y} 2 \_1}$ and $M_{\mathrm{y} 2 \_2}$.

$$
\begin{aligned}
& M_{\mathrm{y} 2_{-1}}=\mathrm{i} \omega \rho \int_{b}^{a} \int_{0}^{2 \pi}\left[\phi_{1}(r,-h, \theta) r^{2} \cos \theta\right] \mathrm{d} r \mathrm{~d} \theta \\
& =2 \pi \mathrm{i} \rho g A \sum_{j=0}^{J} C_{1 j} Y_{j}\left(\lambda_{j}(-h)\right) \int_{b}^{a}\left(V_{m}^{(1)}\left(\lambda_{j} r\right)-\frac{V_{m}^{(1)}{ }^{\prime}\left(\lambda_{j} b\right)}{V_{m}^{(2)}{ }^{\prime}\left(\lambda_{j} b\right)} V_{m}^{(2)}\left(\lambda_{j} r\right)\right) r^{2} \mathrm{~d} r \\
& =2 \pi \mathrm{i} \rho g A\left\{\begin{array}{l}
C_{10} Y_{0}\left(\lambda_{0}(-h)\right) \int_{b}^{a}\left(\mathrm{H}_{1}^{(1)}\left(\lambda_{0} r\right) / \mathrm{H}_{1}^{(1)}\left(\lambda_{0} a\right)-\frac{V_{1}^{(1)}{ }^{\prime}\left(\lambda_{0} b\right)}{V_{1}^{(2)}{ }^{\prime}\left(\lambda_{0} b\right)} \mathrm{H}_{1}^{(2)}\left(\lambda_{0} r\right) / \mathrm{H}_{1}^{(2)}\left(\lambda_{0} a\right)\right) r^{2} \mathrm{~d} r \\
+\sum_{j=1}^{J} C_{1 j} Y_{j}\left(\lambda_{j}(-h)\right) \int_{b}^{a}\left(\mathrm{I}_{1}\left(\lambda_{j} r\right) / \mathrm{I}_{1}\left(\lambda_{j} a\right)-\frac{V_{1}^{(1)}{ }^{\prime}\left(\lambda_{j} b\right)}{V_{1}^{(2)}{ }^{\prime}\left(\lambda_{j} b\right)} \mathrm{K}_{1}\left(\lambda_{j} r\right) / \mathrm{K}_{1}\left(\lambda_{j} a\right)\right) r^{2} \mathrm{~d} r
\end{array}\right\} \\
& =2 \pi \mathrm{i} \rho g A\left\{\begin{array}{l}
C_{10} Y_{0}\left(\lambda_{0}(-h)\right)\left(\frac{a^{2} \mathrm{H}_{2}^{(1)}\left(\lambda_{0} a\right)-b^{2} \mathrm{H}_{2}^{(1)}\left(\lambda_{0} b\right)}{\lambda_{0} \mathrm{H}_{1}^{(1)}\left(\lambda_{0} a\right)}-\frac{V_{1}^{(1)}{ }^{\prime}\left(\lambda_{0} b\right)}{\left.V_{1}^{(2)}\right)^{\prime}\left(\lambda_{0} b\right)} \frac{a^{2} \mathrm{H}_{2}^{(2)}\left(\lambda_{0} a\right)-b^{2} \mathrm{H}_{2}^{(2)}\left(\lambda_{0} b\right)}{\lambda_{0} \mathrm{H}_{1}^{(2)}\left(\lambda_{0} a\right)}\right) \\
+\sum_{j=1}^{J} C_{1 j} Y_{j}\left(\lambda_{j}(-h)\right)\left(\frac{a^{2} \mathrm{I}_{2}\left(\lambda_{j} a\right)-b^{2} \mathrm{I}_{2}\left(\lambda_{j} b\right)}{\lambda_{j} \mathrm{I}_{1}\left(\lambda_{j} a\right)}-\frac{V_{1}^{(1)}{ }^{\prime}\left(\lambda_{j} b\right)}{V_{1}^{(2)}{ }^{\prime}\left(\lambda_{j} b\right)} \frac{a^{2} \mathrm{~K}_{2}\left(\lambda_{j} a\right)-b^{2} \mathrm{~K}_{2}\left(\lambda_{j} b\right)}{\lambda_{j} \mathrm{~K}_{1}\left(\lambda_{j} a\right)}\right)
\end{array}\right\} \\
& M_{\mathrm{y} 2 \_2}=-\mathrm{i} \omega \rho\left[\int_{0}^{a} \int_{0}^{2 \pi} \phi_{3}\left(r,-h_{1}, \theta\right) r^{2} \cos \theta \mathrm{d} r \mathrm{~d} \theta\right] \\
& =-2 \pi \mathrm{i} \rho g A \sum_{j=0}^{J} B_{1 j} Q_{j}\left(\mu_{j}\left(-h_{1}\right)\right) \int_{0}^{a} W_{1}\left(\mu_{j} r\right) r^{2} \mathrm{~d} r \\
& =-2 \pi \mathrm{i} \rho g A\left\{B_{10} Q_{0}\left(\mu_{0}\left(-h_{1}\right)\right) \frac{a^{3}}{4}+\sum_{j=1}^{J} B_{1 j} Q_{j}\left(\mu_{j}\left(-h_{1}\right)\right) \frac{a^{2} I_{2}^{(1)}\left(\mu_{j} a\right)}{\lambda_{j} I_{1}^{(1)}\left(\mu_{j} a\right)}\right\}
\end{aligned}
$$

\section{Results and discussions}

\subsection{Validation}

To validate the analytical model developed in the present study, the wave loads on the structures were compared with the published results for some special cases. Note that excellent convergence can be achieved by truncating the expansion series to the first 20 terms, then 20 terms was adopted throughout the calculations (i.e., $I=J=20$ in Eqs. 19-31). In this section the wave exciting forces in the $x$ and $z$ directions and the overturning moment are non-dimensionalized by $\rho \pi g A a^{2}, \rho \pi g A a^{2}$ and $\rho \pi g A a^{3}$, symbolically, $F_{\mathrm{x}} / \rho \pi g A a^{2}, F_{\mathrm{z}} / \rho \pi g A a^{2}$ and 
$M_{\mathrm{y}} / \rho \pi g A a^{3}$, respectively.

Firstly, a bottom-mounted cylinder with an upper porous wall and an inner column was taken as an example, which corresponds to the gap between the cylinder bottom and the sea bed equal to zero in Fig. 1, i.e., $h_{1}=d$. The following parameters $h / h_{1}=0.5, b / a=0.5, a / h_{1}=1, G=$ $0.1+$ i 0.1 (i.e., case 1) and $0.4+$ i 0.4 (i.e., case 2) are chosen. Fig. 2 shows the comparison between the dimensionless horizontal force calculated by the present model and another analytical solution by Teng et al. (2001) for the bottom-mounted cylinder with an upper porous outside-wall. The horizontal axis represents the dimensionless wave number $\mathrm{ka}$. From the figure, it can be seen that a good agreement between the two methods is observed from the comparison.

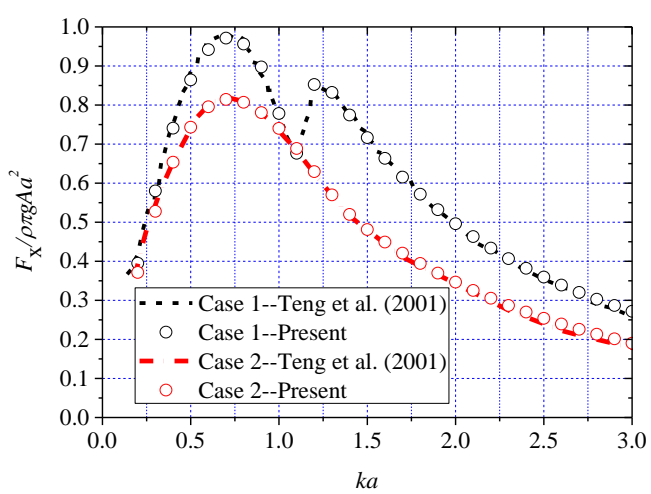

Figure 2 Comparison of dimensionless horizontal forces obtained from the present model and those from Teng et al. (2001)

Secondly, the case of a bottom-mounted compound cylinder was considered for verification, which corresponds to the case of $h_{1}=d$ in Fig. 1. The following parameters $h / h_{1}=0.5, h_{1} / a=2$, $G=1 \mathrm{E} 10+\mathrm{i} 1 \mathrm{E} 10, b / a=0.5$ (i.e., case 1) and 0.75 (i.e., case 2) are chosen. Fig. 3 shows the comparison of the dimensionless horizontal force acting on the base cylinder (symbolically, $\left.F_{\mathrm{x}, \mathrm{b}} / \rho \pi g A a^{2}\right)$ between the present results and the corresponding solution by Isaacson (1979). From the figure, it can be seen that a good agreement is obtained. 


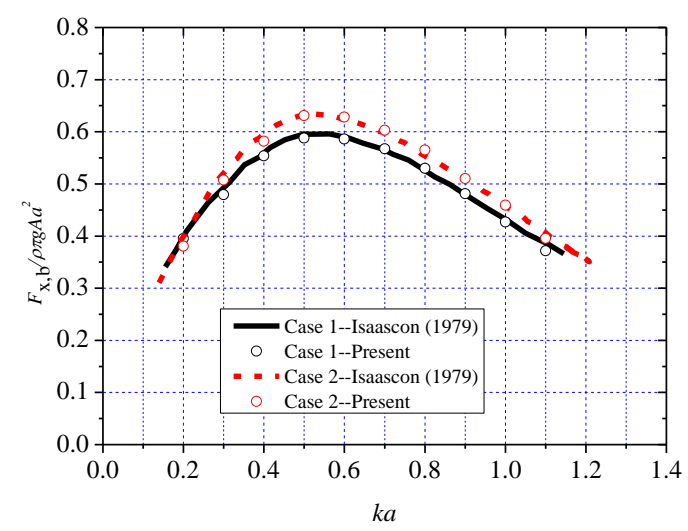

Figure 3 Comparison of dimensionless horizontal force obtained from the present model and that from Isaacson (1979)

Thirdly, a truncated impermeable cylinder was chosen. The following parameters $b / a=0.5$, $h_{1}=a, h_{1} / d=2 / 3$ and $G=0$ are chosen. Fig. 4 shows the comparison between the dimensionless wave forces/moment $\left(F_{\mathrm{x}} / \rho \pi g A a^{2}, F_{\mathrm{z}} / \rho \pi g A a^{2}\right.$ and $\left.M_{\mathrm{y}} / \rho \pi g A a^{3}\right)$ obtained from the present model and the results from Garrett (1971). The analytical method based on linear potential theory is adopted by Garrett (1971). The moment center is at the structure base, i.e., $\left(0,0,-h_{1}\right)$. It can be seen that all the exciting forces agree very well with the corresponding results of Garrett (1971).

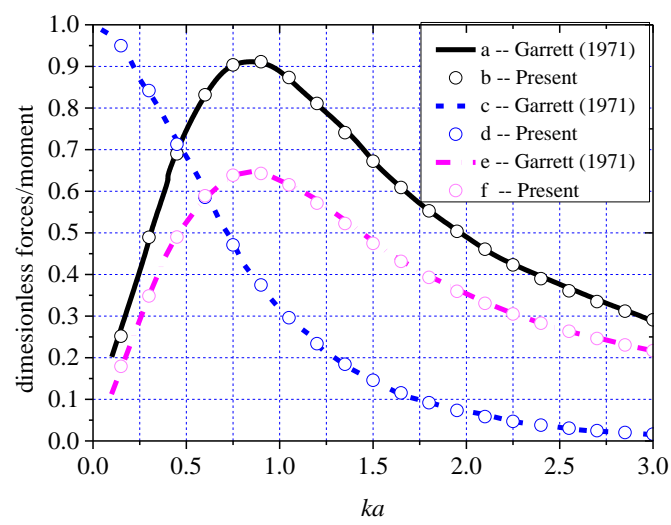

Figure 4 Comparison of the dimensionless wave forces/moment obtained from the present model and the results from Garrett (1971). (a, b) $F_{\mathrm{x}} / \rho \pi g A a^{2}$; (c, d) $F_{\mathrm{z}} / \rho \pi g A a^{2}$; (e, f) $M_{\mathrm{y}} / \rho \pi g A a^{3}$.

At last, a truncated compound cylinder was used to validate the present formulation, i.e., the porous parameter $G$ was chosen as an infinite value in the present study. The following parameters $d / a=6, b / a=2, h_{1} / h=4, h_{1} / a=3$ and $G=1 \mathrm{E} 10+\mathrm{i} 1 \mathrm{E} 10$ are chosen. Fig. 5 shows the comparison between the dimensionless wave forces/moment $\left(F_{\mathrm{x}} / \rho \pi g A a^{2}, F_{\mathrm{z}} / \rho \pi g A a^{2}\right.$ and $M_{\mathrm{y}} / \rho \pi g A a^{3}$ ) obtained from the present model and the results from Kokkinowrachos et al. (1986). 
The analytical method based on linear potential theory is adopted by Kokkinowrachos et al. (1986). The moment center is at the structure base, i.e., $\left(0,0,-h_{1}\right)$. It can be seen that all the exciting forces agree very well with the corresponding results of Kokkinowrachos et al. (1986).

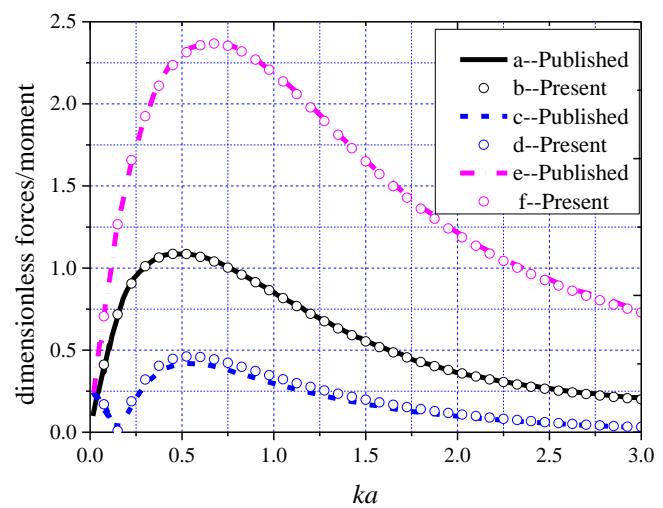

Figure 5 Comparison of the dimensionless wave forces/moment obtained from the present model and the results from Kokkinowrachos et al. (1986). (a, b) $F_{\mathrm{x}} / \rho \pi g A a^{2}$; (c, d) $F_{\mathrm{z}} / \rho \pi g A a^{2}$; (e, f) $M_{\mathrm{y}} / \rho \pi g a^{3}$.

Overall, the four comparisons with good agreement demonstrate the accuracy of the present analytical model.

\subsection{Parametric study}

Following the above model validation, the parametric studies were carried out to determine the influence of the different characteristics (porous coefficient $G$, draft ratio $h / h_{1}$, radius ratio $b / a$ and water depth $d / h_{1}$ ) on the wave forces acted on the structure. The moment center is taken at the static waterline, i.e., at $(0,0,0)$.

\subsubsection{Effect of the imaginary part of porous coefficient $G$}

The real value of porous coefficient $G$ is often used to investigate the effect of porosity on the hydrodynamics of the structures. It is understood that the real part of the porous coefficient has a significant influence on the hydrodynamic loads acted on the porous structure (Williams et al., 2000; Lee and Ker, 2002; Teng et al., 2000). As mentioned, both the real part and the imaginary part of the porous coefficient denote the physical significance. Teng et al.'s (2001) 
results showed that the imaginary part of the $G$ affects the wave force acting on the bottom-mounted porous cylinder. So it is meaningful to investigate the effect of the imaginary part of the porous coefficient on the hydrodynamic forces acting on the structure. In this section, the parameters $b / a=0.5, d / h_{1}=2, h / h_{1}=0.5$ and $h_{1}=a$ were fixed and numerical calculations were performed with four porous coefficients of $G=1+\mathrm{i} 0,1+\mathrm{i} 0.5,1+\mathrm{i} 1$ and $1+\mathrm{i} 1.5$ to investigate the effect of the imaginary part of the $G$ on the hydrodynamic forces $\left(F_{\mathrm{x}}, F_{\mathrm{z}}, M_{\mathrm{y}}\right)$.

From the Figs. 6(a-c), it can be observed that all curves in each figure keep the similar trend. For the Fig. 6(a) and 6(c), the remarkable influence of the imaginary part of the $G$ can be found in the relative high frequency region $(k a>0.8$, approximately), where the horizontal force decreases and the moment increases with the increase of the imaginary part of the $G$. By contrast, the vertical force is more sensitive to the imaginary part of the $G$ over the whole range of frequencies. With the increase of the imaginary part of the $G$, the vertical force decreases in the lower frequency region and the vertical force increases in the higher frequency region. This may be due to the fact that the imaginary part of $G$ mainly lead to the phase change and the wave forces is associated with the phase angle of the neighboring waves.
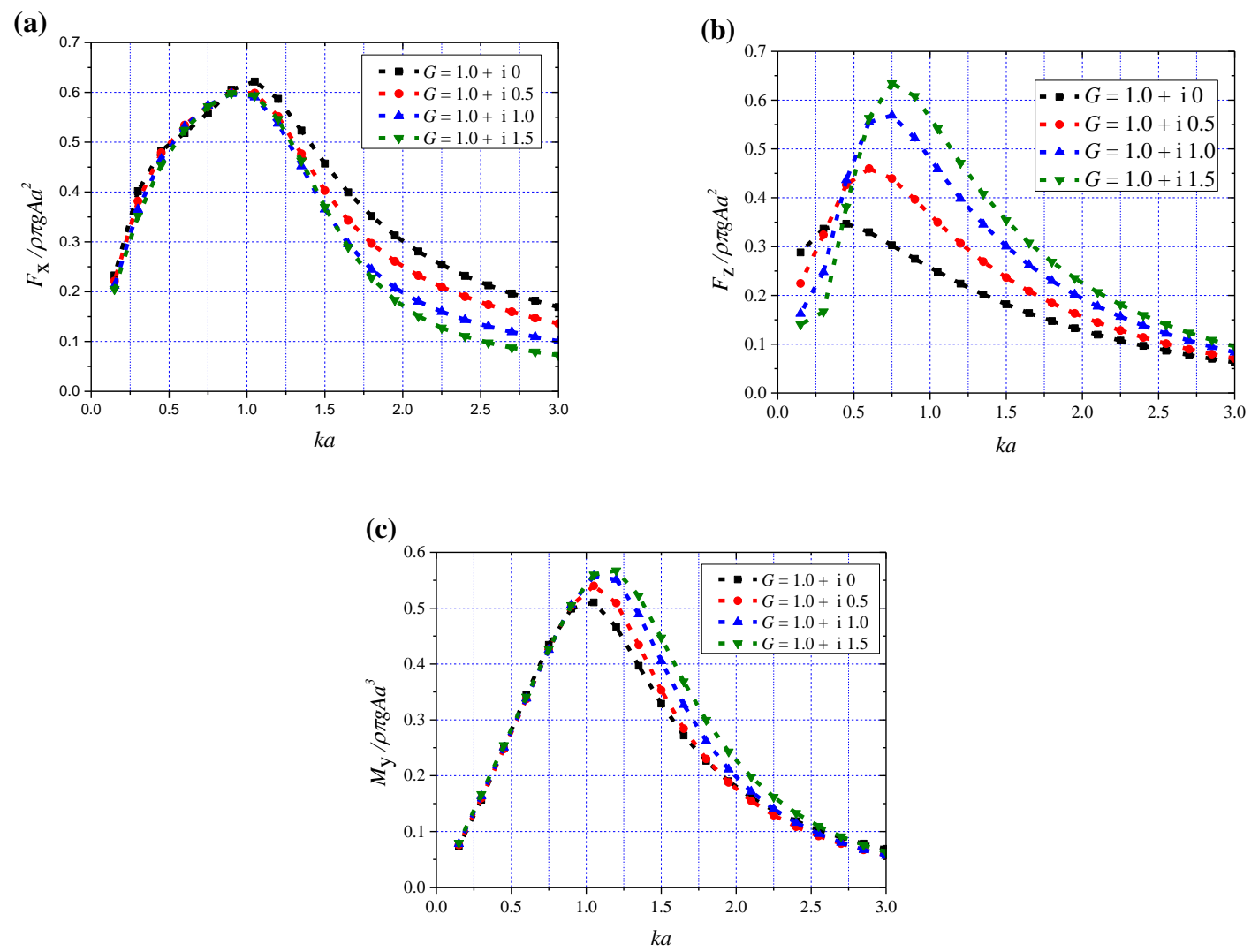

Figure 6 The dimensionless wave forces/moment $v s$ the dimensionless wave number $k a$ for the different $G$. (a) $F_{\mathrm{x}} / \rho \pi g A a^{2}$; (b) $F_{\mathrm{z}} / \rho \pi g A a^{2}$; (c) $M_{\mathrm{y}} / \rho \pi g A a^{3}$. 


\subsubsection{Effect of draft ratio $\left(h / h_{1}\right)$}

The influence of the porous coefficient on the hydrodynamic forces $\left(F_{\mathrm{x}}, F_{\mathrm{z}}, M_{\mathrm{y}}\right)$ under different draft ratio $\left(h / h_{1}\right)$ is of interest. The parameters $b / a=0.5, d / h_{1}=2$ and $h_{1}=a$ are fixed. Numerical calculations were performed with four porous coefficients of $G=0,0.5+\mathrm{i} 0.5,1+\mathrm{i}$ 1 and $1.5+\mathrm{i} 1.5$, and three draft ratios of $h / h_{1}=0.25,0.5$ and 0.75 , respectively. Note that, to better reflect the realistic role of the porous structures, the complex from of $G\left(G_{\mathrm{r}}=G_{\mathrm{i}}\right.$ in each case) is chosen to conduct the parametric study.

From the Figs. 7(a-c), it can be observed that all curve shapes keep the similar trend (i.e., increases firstly and then decreases with the increase of $k a$ ) for different parameters $G$ and $h / h_{1}$. Obviously, the maximum horizontal wave load occurs at $G=0$, i.e., the impermeable structure. Both porous coefficient and draft of porous part can make the horizontal wave load reduced. However, the horizontal wave loads reach to a saturation status when the porous parameter $G$ increases to a certain value for deeper draft of porous part (i.e., $h / h_{1}=0.5$ and 0.75 ). 

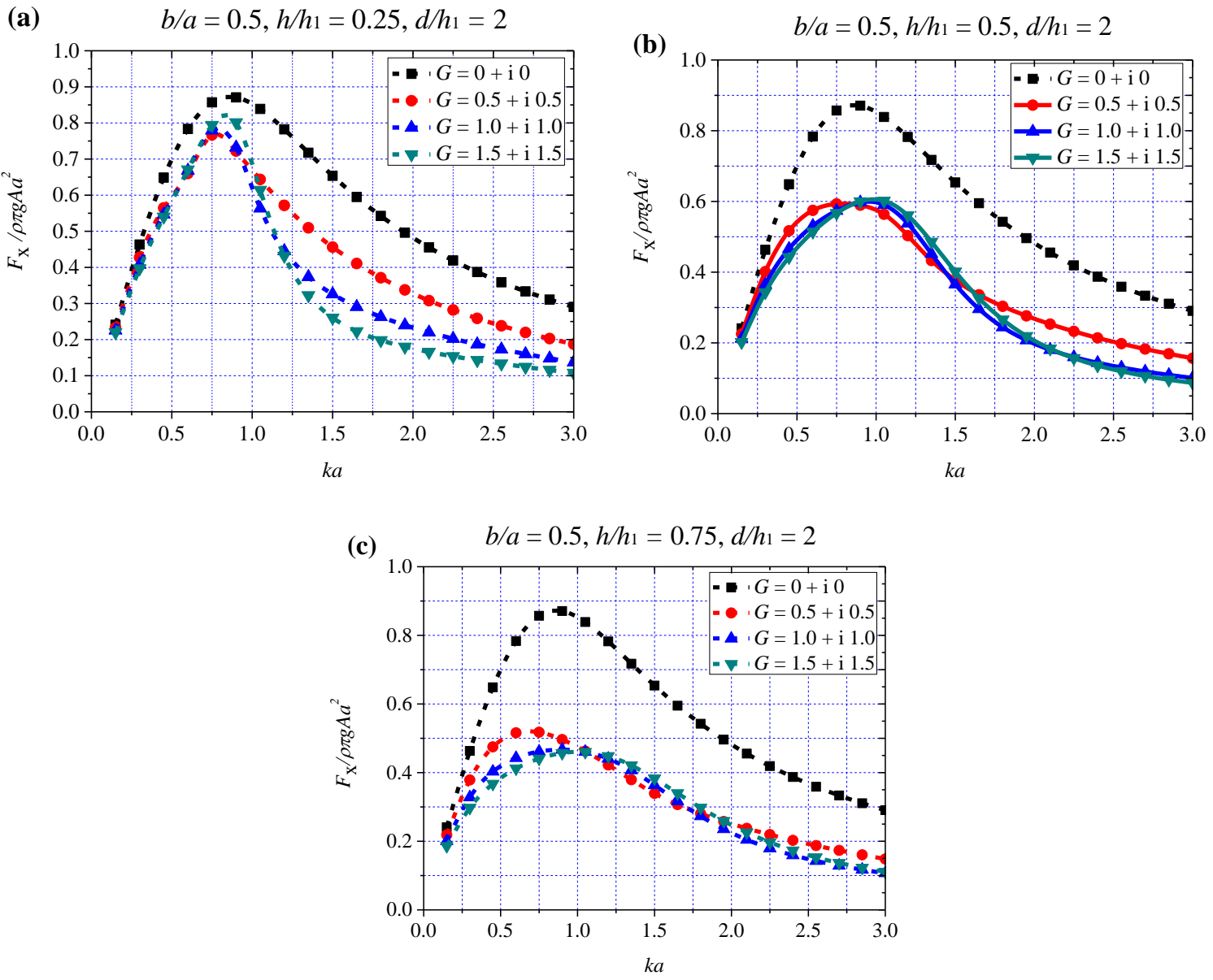

Figure 7 Variation of $F_{\mathrm{x}} / \rho \pi g A a^{2} v s$ the dimensionless wave number $k a$ for different $h / h_{1}$

Fig. 8 shows the variation of dimensionless vertical wave loads $F_{\mathrm{z}} / \rho \pi g A a^{2}$ on the structure versus the dimensionless wave number $k a$ for the different porous and draft parameters. As a comparison, the results for the impermeable truncated cylinder (i.e., $G=0$ ) are also plotted in the figures. The vertical wave loads on the impermeable truncated cylinder monotonically decreases with $k a$, which is due to the fact that the velocity potential near the outer bottom (i.e., $\left.S_{\mathrm{h} 2}\right)$ decreases with the decrease of wave length. Different from the impermeable case, there exists a critical wave frequency $f_{c}$, and the vertical force on the porous structure is smaller than that on the impermeable structure if $f<f_{c}$. Oppositely, the vertical force on the porous structure is larger if $f>f_{c}$. The critical frequency $f_{c}$ increases with the increase of porous parameter $G$. Besides, the vertical force on the porous structure reaches the maximum at another certain frequency (i.e., resonant frequency) and then decreases towards the longer and shorter waves. This may be due to the phase difference of vertical forces on the inner and outer bottoms. Similar to the conclusions obtained in Fig. 7, the vertical wave force on the structure also 
decreases with the increase of porous coefficient in the low frequency region; it is completely opposite in the high frequency region. The vertical wave force decreases with the increase of the draft of the porous part. Specially, there exist trough vertical forces acting on the structure for cases of larger $G$ and $h / h_{1}$ as shown in Fig. 8 (b) and (c). Similar phenomenon was ever found by Miao and Liu (1987) in the study of wave diffraction from a compound cylinder (i.e., a column with footing), for which the trapped wave may be induced at some special wave frequencies. The structure in this study is near to the compound cylinder while $G$ is large enough. Besides, the vertical wave force tends to saturation with the increase of $G$ in the high frequency region, especially for larger $h / h_{1}$.
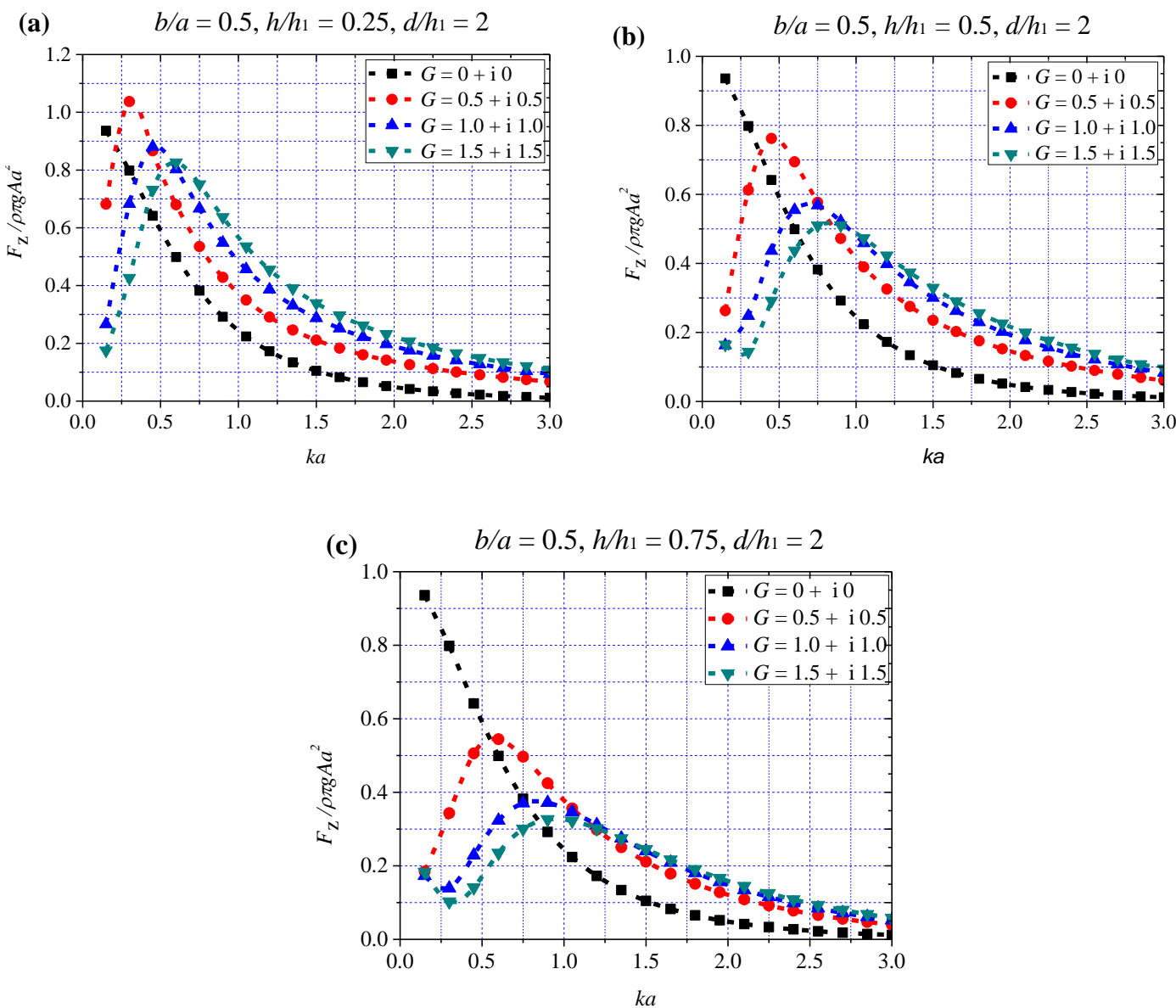

Figure 8 Variation of $F_{\mathrm{z}} / \rho \pi g A a^{2} v s$ the dimensionless wave number $k a$ for different $h / h_{1}$

Fig. 9 shows the variations of dimensionless wave moment $M_{\mathrm{y}} / \rho \pi g A a^{3}$ along $y$-axis versus dimensionless wave number $k a$ with different parameters $\left(G\right.$ and $\left.h / h_{1}\right)$. The moment center is at $(0,0,0)$. Different from the variations of horizontal and vertical wave forces on the structure 
shown in Figs. 7 and 8, the peak moment for cases of $G$ being nonzero is greater than that of $G=$ 0 . This may be related to the fact that the horizontal forces on the inner and outer cylinders focus on the upper and lower parts, respectively, considering the phase difference between these two forces, which leads to a larger moment than that on the impermeable cylinder. However, it decreases with the increase of the draft of the porous part, which may be attributed to the decreasing of horizontal wave loads on the structure. The moment changes little with the increase of $G$ while the structure is under action of longer waves. Correspondingly, the horizontal force follows this principle and the vertical force does not. From this point, it can also be conclude that the horizontal force may dominate the contribution to the moment. Besides, for the cases of deeper $h / h_{1}$, the wave moment is easy to reach to a saturation status with the increase of $G$.
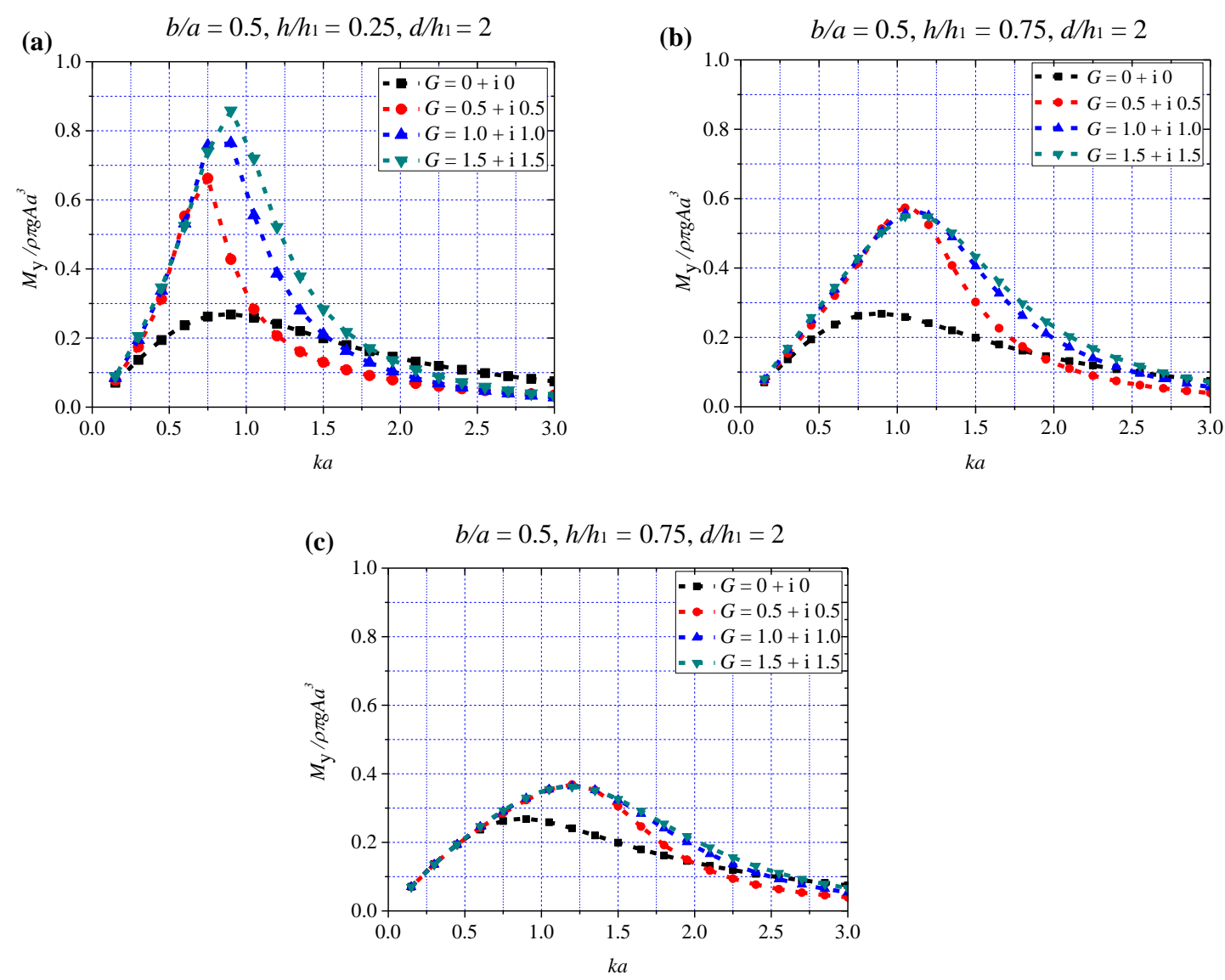

Figure 9 Variation of $M_{\mathrm{y}} / \rho \pi g A a^{3}$ vs the dimensionless wave number $k a$ for different $h / h_{1}$

\subsubsection{Effect of the ratio of the inner and outer radii $(b / a)$}

In this section, the influence of porous coefficient on the hydrodynamic forces $\left(F_{\mathrm{x}}, F_{\mathrm{z}}, M_{\mathrm{y}}\right)$ 
with different ratio of the inner and outer radii $(b / a)$ was investigated. The parameters $h / h_{1}=0.5$, $d / h_{1}=2$ and $h_{1}=a$ are fixed. Four porous coefficients (i.e., $G=0,0.5+\mathrm{i} 0.5,1+\mathrm{i} 1$ and $1.5+\mathrm{i}$ $1.5)$ were adopted for each group. Three cases of $b / a(0.25,0.5$ and 0.75$)$ were selected.

Fig. 10(a), Fig. 7(b) and Fig. 10(b) show the variations of $F_{\mathrm{x}} / \rho \pi g A a^{2}$ versus $k a$ with different ratio of inner and outer radii. Similar to the case of $b / a=0.5$ in Fig. 7(b), the horizontal force of porous structures are reduced effectively by increasing the value of $G$. The horizontal force increases firstly and then decreases with the increase of $k a$. Specially, an oscillation was observed for the case of $G=0.5+$ i 0.5 and $b / a=0.25$ in Fig. 10(a), which may be attributed to the phase difference of the forces acting on the inner column and the outer wall (Teng et al., 2000). By comparing with the Fig. 10(a), Fig. 7(b) and Fig. 10(b), it was observed that the horizontal force reduction is stronger for the cases of smaller $b / a$ while the porous structure plays the same role. Besides, the variation of $F_{\mathrm{x}} / \rho \pi g A a^{2}$ is more near to that of the impermeable cylinder and is easy to reach a saturation status with the increase of the $G$. This may be due to the fact that the structure is near to the impermeable cylinder geometrically for larger inner radius $(b / a=0.75)$.

(a)

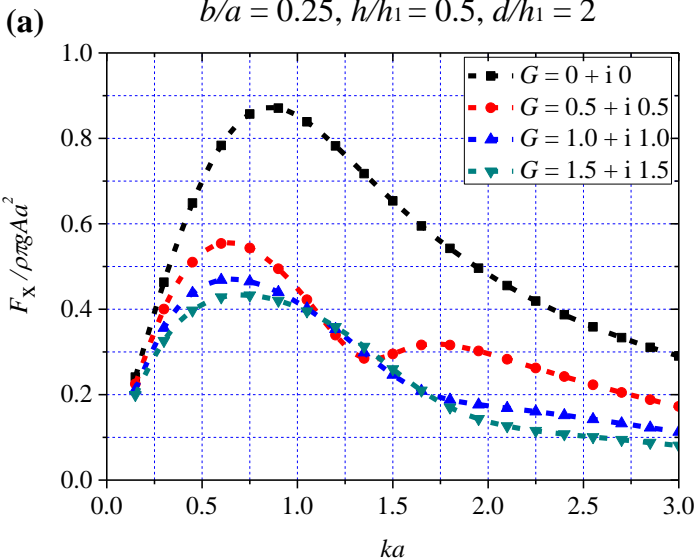

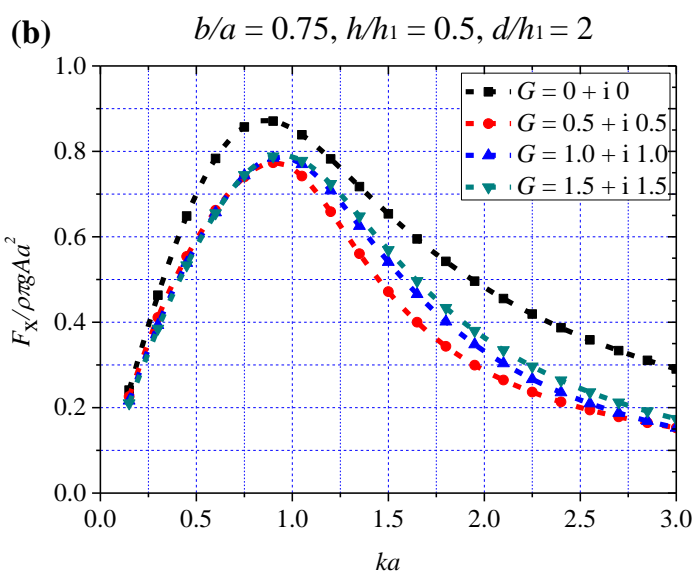

Figure 10 Variations of $F_{\mathrm{x}} / \rho \pi g A a^{2} v s$ the dimensionless wave number $k a$ for different $b / a$

Fig. 11(a), Fig. 8(b) and Fig. 11(b) show the variations of $F_{z} / \rho \pi g A a^{2}$ versus $k a$ for different $b / a$. The variation of $F_{z} / \rho \pi g A a^{2}$ for the case of $b / a=0.25$ in Fig. 11(a) is similar to that for $b / a=$ 0.5 in Fig. 8(b). The difference is that, for a certain porous coefficient $G$, the peak value of $F_{\mathrm{z}} / \rho \pi g A a^{2}$ increases and the so-called critical frequency $f_{\mathrm{c}}$ decreases. And for the case of $b / a=$ 
0.75 , the variation of $F_{z} / \rho \pi g A a^{2}$ is near to that of the impermeable one due to the porous structure near to the impermeable cylinder geometrically as shown in Fig. 11(b).
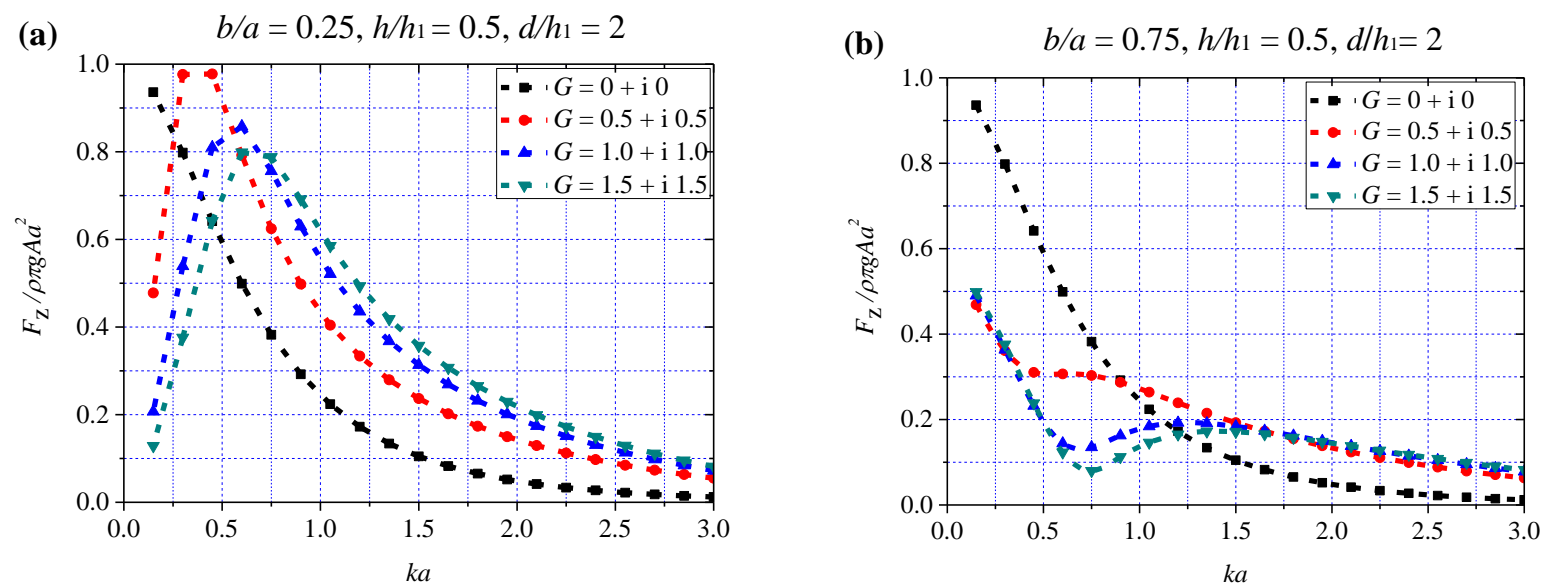

Figure 11 Variation of $F_{\mathrm{z}} / \rho \pi g A a^{2} v s$ the dimensionless wave number $k a$ for different $b / a$

Fig. 12(a), Fig. 9(b) and Fig. 12(b) show the variations of $M_{\mathrm{y}} / \rho \pi g A a^{3}$ versus the dimensionless wave number $k a$ for different $b / a$. Similar to Fig. 9(b), the moment changes little for different porous coefficient $G$ in the low frequency region, which may be due to the strong transmission ability for long waves. Such phenomenon is especially apparent for smaller $b / a$, because the horizontal force on the inner cylinder decreases and the vertical force on the upper bottom increases, which both make the moment $M_{y}$ decreasing. The difference of the moment $M_{y}$ among different porous coefficient $G$ decreases with the increase of $b / a$ in the high frequency region. However, the moments $M_{y}$ for the porous structure are much larger than that for the impermeable one in the resonant region. The resonant frequency shifts to a smaller value for a larger radius ratio $b / a$. Furthermore, the resonant frequency for the case of $b / a=0.75$ is near to that for the impermeable structure.
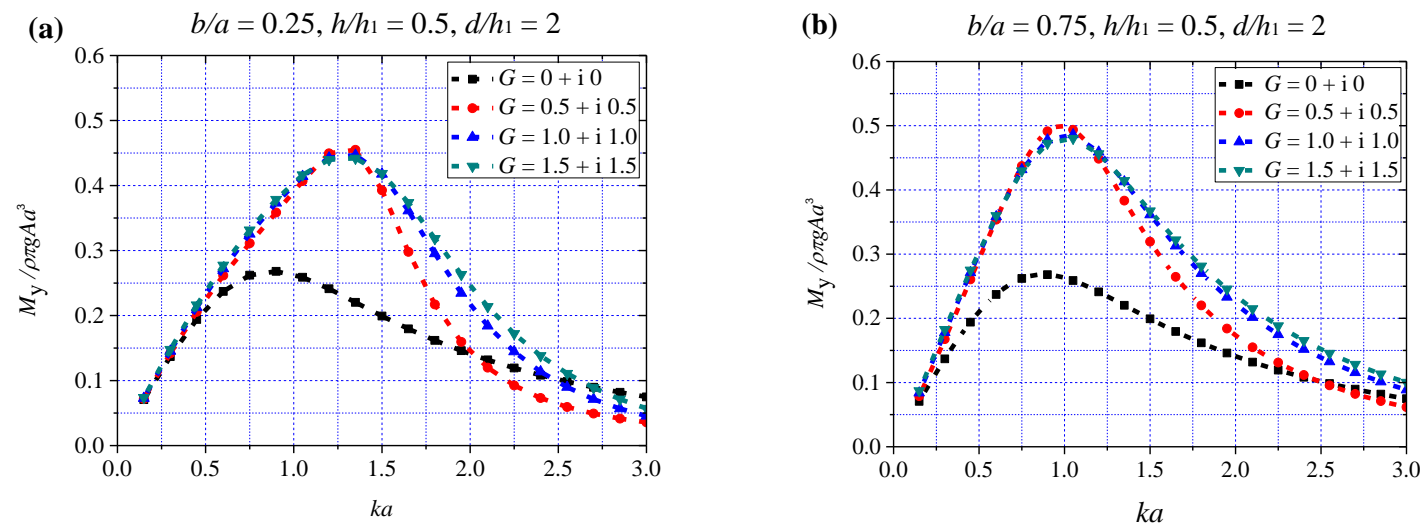


\subsubsection{Effect of the water depth $\left(d / h_{1}\right)$}

In this section, the influence of porous coefficient on the excitation forces/moment for different ratios of inner and outer water depth (i.e., $d / h_{1}=1.5,2$ and 4 ) is considered. In the computation, the parameters $b / a=0.5, h / h_{1}=0.5$ and $h_{1}=a$ are fixed. Four porous coefficients, i.e., $G=0,0.5+\mathrm{i} 0.5,1+\mathrm{i} 1$ and $1.5+\mathrm{i} 1.5$, were chosen.

Fig. 13(a), Fig. 7(b) and Fig. 13(b) show the variations of the dimensionless horizontal forces versus wave number $k a$ for different water depths. By comparing these three figures, it can be seen that, for a fixed $G$, the peak value of $F_{\mathrm{x}} / \rho \pi g A a^{2}$ decreases slightly with the increase of water depth $d / h_{1}$. The difference of the horizontal force among different porous structures decreases with the increase of water depth. Generally, the water depth has a little influence on the horizontal force.
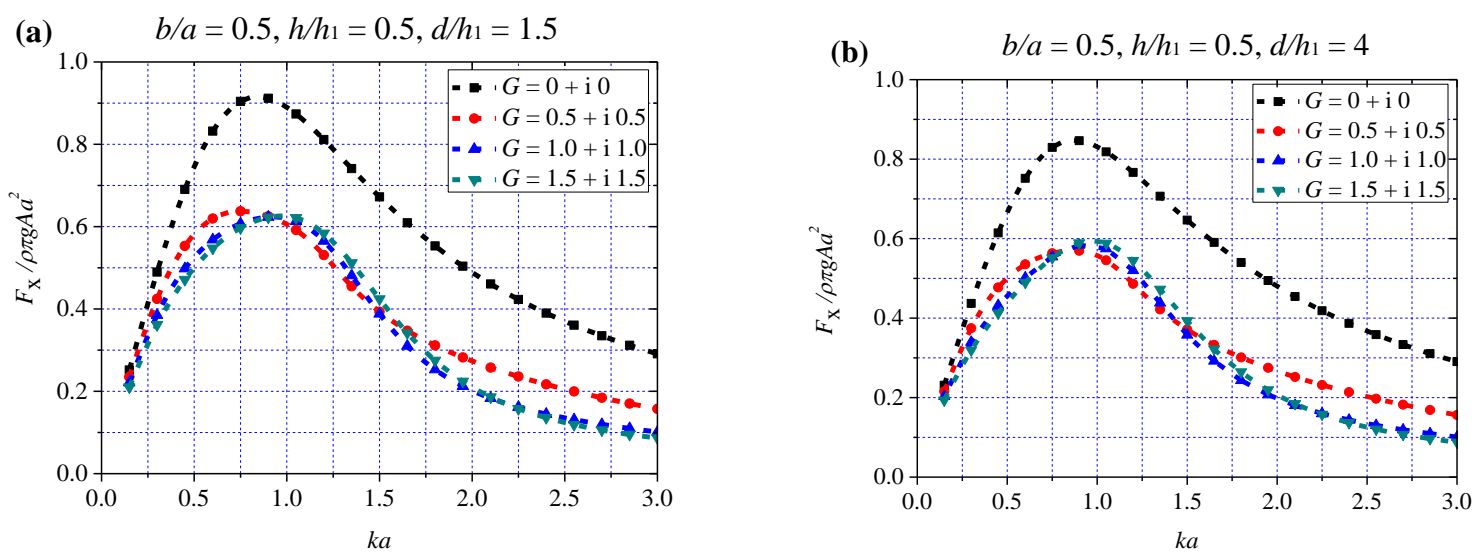

Figure 13 Variation of $F_{\mathrm{x}} / \rho \pi g A a^{2} v s$ the dimensionless wave number $k a$ for different $d / h_{1}$

Fig. 14(a), Fig. 8(b), and Fig. 14(b) show the variations of vertical forces versus $k a$ for three water depths. From the figures, it can be seen that the water depth significantly influences the vertical force, especially in the low frequency region. The peak vertical force increases but the critical wave frequency $f_{\mathrm{c}}$ decreases with the increase of the water depth. For the same $k a$, the excitation frequency increases with the water depth increasing. Thus the high frequency wave makes greater contribution on the vertical wave loads on the porous structure. Similar to the phenomena shown in Figs. 8(b-c), the trough vertical force on the porous structure can be found 
in the relative shallow water in Fig. 14(a).

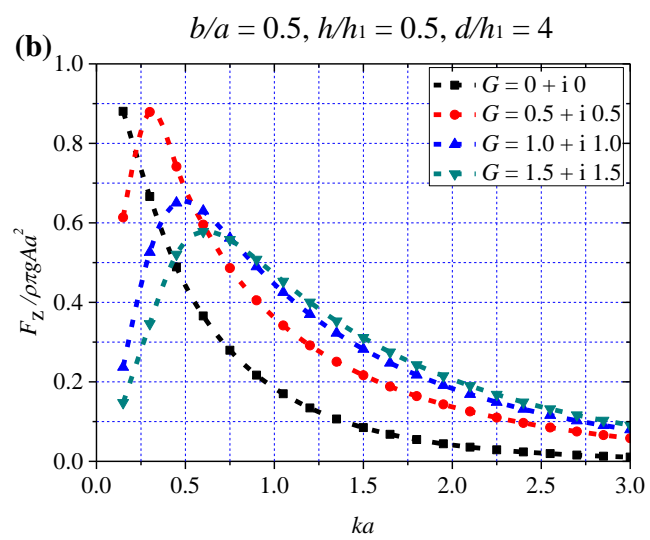

Figure 14 Variation of $F_{\mathrm{z}} / \rho \pi g A a^{2} v s$ the dimensionless wave number $k a$ for different $d / h_{1}$

Fig. 15(a), Fig. 9(b) and Fig. 15(b) show the variations of $M_{\mathrm{y}} / \rho \pi g A a^{3}$ versus $k a$ for different water depths. By comparing Figs. (13-15), it can be seen that the moment $M_{y}$ shows a similar variation as the horizontal force. They both are less influenced by the water depth. Due to the phase difference of the horizontal wave loads on the inner and outer cylinders (Teng et al., 2000), the horizontal wave force on the porous structure is smaller than that acted on the impermeable one, but it is completely opposite for the moment $M_{y}$.
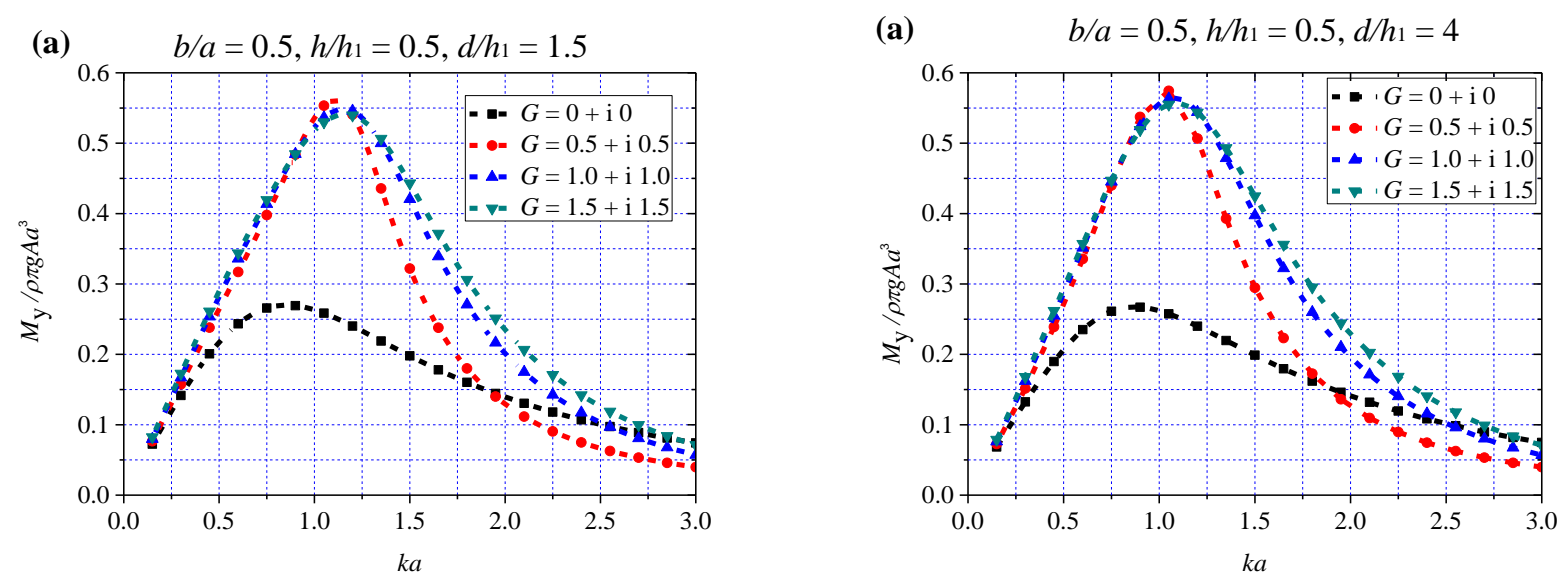

Figure 15 Variation of $M_{\mathrm{y}} / \rho \pi g A a^{3}$ vs the dimensionless wave number $k a$ for different $d / h_{1}$

\section{Conclusions}

Based on the linear water wave theory, the diffraction problem was dealt with for a truncated cylinder with the upper part comprising a porous sidewall and an inner column. The velocity potential was analytically derived in the whole fluid domain based on the method of 
variable separation and eigen-function expansion technique. By using the analytical expressions, the wave forces are derived and calculated. Then the effects of the various parameters on the hydrodynamic properties of the structure were investigated, which may provide some useful information for the relating design.

Numerical tests show that the porous coefficient $G$ affects the wave loads on the structure apparently. Compared with the impermeable truncated cylinder, the horizontal force on the cylinder with a porous structure like Fig. 1 is reduced effectively. The vertical force is reduced in the low frequency region but increased in the high frequency region. Generally, the peak moment around $y$ axis increases while the porous structure plays a role. Besides, the parameters (such as draft ratio $h / h_{1}$, ratio of inner and outer radius $b / a$ and water depth $d / h_{1}$ ) are also the key factors affecting wave loads on the structure. The wave forces/moments decrease with the increase of the draft ratio; the variations of the wave forces/moments are near to those of the impermeable structure while the ratio of inner and outer radius approaches to 1; the water depth significantly influences the vertical force, but relatively slightly for the horizontal force and the overturning moment.

In a word, the proposed structure with proper parameters $\left(G, h / h_{1}, b / a\right.$ and $\left.d / h_{1}\right)$ can be used to decrease the surge wave force and the heave force acting on the structure, especially in the long waves.

\section{Acknowledgements:}

The authors would like to gratefully acknowledge the financial support from the National Science Foundation of China (Grant Nos. 51222902, 51490672) and the Program for New Century Excellent Talents in University (Grant No.NCET-13-0076).

\section{Reference}

Chandrasekaran, S., Madhavi, N., Sampath, S., 2015. Force reduction on ocean structures with perforated members. Adv. in Struct. Eng. Springer, 647-661.

Chandrasekaran, S., Sharma, A., 2010. Potential-flow-based numerical study for the response of floating offshore structures with perforated columns. Ships Offshore Struct. 5(4), 327-336. 
Chwang, A. T, Chan, A. T., 2003. Interaction between porous media and wave motion. Annu. Rev. Fluid Mech. 30(5), 53-84.

Faizal, M., Ahmed, M.R., Lee, Y.-H., 2014. A design outline for floating point absorber wave energy converters. Adv. in Mech. Eng. 2014, 1-18.

Garrett, C.J.R., 1971. Wave forces on a circular dock. J. Fluid Mech. 46(01), 129-139.

Göteman, M., Engström, J., Eriksson, M., Isberg, J., Leijon M., 2014. Analytical and numerical approaches to optimizing fluid-structure interactions in wave energy parks. Proceedings of the 29th International Workshop on Water Waves and Floating Bodies (IWWWFB), Osaka, Japan.

Hirdaris, S., Bai, W., Dessi, D., Ergin, A., Gu, X., Hermundstad, O., Huijsmans, R., Iijima, K., Nielsen, U.D., Parunov, J., 2014. Loads for use in the design of ships and offshore structures. Ocean Eng. 78, 131-174.

Hu, H., Wang, K.H., Williams, A.N., 2002. Wave motion over a breakwater system of a horizontal plate and a vertical porous wall. Ocean Eng. 29(4), 373-386.

Huang, Z.H., Li, Y.C., Liu, Y., 2011. Hydraulic performance and wave loadings of perforated/slotted coastal structures: a review. Ocean Eng. 38(10), 1031-1053.

Isaacson, M. (1979). Wave forces on compound cylinders. Proceedings of Civil Engineering in the Oceans IV, ASCE, San Francisco, 518-530.

Jarlan, G., (1961). A perforated vertical wall breakwater. Dock Harbour Auth. 41(486), 394-398.

Jiang, S.C., Gou, Y., Teng, B., Ning, D.Z., 2014. Analytical solution of a wave diffraction problem on a submerged cylinder. J. Eng. Mech. 140(1), 225-232.

Johanning, L., 2009. Dynamic response characteristics of a floating wind turbine tower at low response frequency. Proceedings of International Conference on Ocean, Offshore and Arctic Engineering - OMAE, 4part B: 983-992.

Johanning, L., Bearman, P. W., Graham, J.M.R., 2001. Hydrodynamic damping of a large scale surface piercing circular cylinder in planar oscillatory motion. J. Fluids Struct. 15(7), 891-908.

Kara, F., 2016. Time domain prediction of power absorption from ocean waves with wave energy converter arrays. Renew. Energy 92, 30-46.

Kokkinowrachos, K., Mavrakos, S., Asorakos, S., 1986. Behaviour of vertical bodies of 
revolution in waves. Ocean Eng. 13(6), 505-538.

Lee, C.-P., Ker, W.-K., 2002. Coupling of linear waves and a hybrid porous TLP. Ocean Eng. 29(9), 1049-1066.

Li, Y.C., Sun, L., Teng, B., 2004. Wave interaction with an array of combined cylinders with solid interior column and porous exterior column. The 6th ISOPE Pacific/Asia Offshore Mechanics Symposium, International Society of Offshore and Polar Engineers, Russia.

Liu, Y., Li, Y.C., Teng, B., Jiang, J.J., 2006. Experimental and theoretical investigation of wave forces on a partially-perforated caisson breakwater with a rock-filled core. China Ocean Eng. 20(2), 179-198.

Liu, Y., Li, Y.C., Teng, B., Jiang, J.J., Ma, B.L., 2008. Total horizontal and vertical forces of irregular waves on partially perforated caisson breakwaters. Coast Eng. 55(6), 537-552.

Mandal, S., Sahoo, T., (2015). Axisymmetric gravity wave diffraction by flexible porous cylinder system in two-layer fluid. Ocean Eng, 106, 87-101.

Miao, G.P., Liu, Y.Z., 1987. Wave forces on a column with footing[J]. China Ocean Eng. 1(3), $17-26$.

Ning, D.Z., Zhao, X.L., Göteman, M., Kang, H.G., 2016. Hydrodynamic performance of a pile-restrained WEC-type floating breakwater: An experimental study. Renew. Energy 95, $531-541$.

Park, M.S., Jeong, Y.J., You, Y.J., Lee, D.H., Kim, H.C., 2014. Numerical analysis of a gravity substructure with partial porous area for offshore wind turbines. J. Civil Eng. and Architect Res. 1(4): 230-238.

Sankarbabu, K., Sannasiraj, S., Sundar, V., 2007. Interaction of regular waves with a group of dual porous circular cylinders. Appl. Ocean Res. 29(4), 180-190.

Sollitt, C.K., Cross, R.H., 1972. Wave transmission through permeable breakwaters. In: Proceedings of 13th International Conference Coastal Engineering, ASCE, New York, $1847-1865$.

Teng, B., Han, L., Li Y.C., 2000. Ware diffraction from a vertical cylinder with two uniform columns and porous outer wall. China Ocean Eng. 14(3), 297-306.

Teng, B., Zhao, M., Li, Y.C., 2001. Wave diffraction from a cylinder with porous upper wall and an inner column. Acta Oceanolog. Sin. 23(6), 133-142. 
Ursell, F., 1987. Mathematical aspects of trapping modes in the theory of surface waves. J. Fluid Mech. 183(1), 421-437.

Williams, A.N., Li, W., 1998. Wave interaction with a semi-porous cylindrical breakwater mounted on a storage tank. Ocean Eng. 25(2), 195-219.

Williams, A.N., Li, W., 2000. Water wave interaction with an array of bottom-mounted surface-piercing porous cylinders. Ocean Eng. 27(8), 841-866.

Williams, A.N., Li, W., Wang, K.-H., 2000. Water wave interaction with a floating porous cylinder. Ocean Eng. 27(1), 1-28.

Wolgamot, H.A., Taylor, P.H., Taylor, R.E., 2012. The interaction factor and directionality in wave energy arrays. Ocean Eng. 47, 65-73.

Yu, X.P., 1995. Diffraction of water waves by porous breakwaters. J. Waterway, Port, Coastal Ocean Eng. 121(6), 275-282.

Yu, X.P., Chwang, A.T., 1994. Water waves above submerged porous plate. J. Eng. Mech. 120(6), $1270-1282$.

Zheng, S.M., Zhang, Y.L., 2015. Wave diffraction from a truncated cylinder in front of a vertical wall. Ocean Eng 104: 329-343. 